\title{
Detection of $\mathrm{CO}^{+}$toward the reflection nebula NGC 7023
}

\author{
A. Fuente and J. Martín-Pintado \\ Observatorio Astronómico Nacional (IGN), Campus Universitario, Apdo. 1143, E-28800 \\ Alcalá de Henares (Madrid), Spain
}

Received — 


\begin{abstract}
We have detected $\mathrm{CO}^{+}$toward the photon-dominated region (PDR) associated with the reflection nebula NGC 7023. This is the first detection of $\mathrm{CO}^{+}$in the vicinity of a Be star. $\mathrm{A} \mathrm{CO}^{+}$column density of $\sim 310^{11} \mathrm{~cm}^{-2}$ has been derived toward the PDR peak. We have, however, not detected $\mathrm{CO}^{+}$in a well shielded clump of the adjacent molecular cloud, where the $\mathrm{CO}^{+} / \mathrm{HCO}^{+}$ abundance ratio is at least 100 times lower than in the PDR. Our results show, for the first time, that $\mathrm{CO}^{+}$column densities as large as $\sim 310^{11} \mathrm{~cm}^{-2}$ can be produced in regions with incident UV fields of just a few $10^{3}$ (in units of Habing field) and densities of $\leq 10^{5} \mathrm{~cm}^{-3}$. Furthermore, since the ionization potential of $\mathrm{CO}$ is larger than $13.6 \mathrm{eV}$, our data rule out the direct photoionization of $\mathrm{CO}$ as a significant $\mathrm{CO}^{+}$formation mechanism.
\end{abstract}

Subject headings: ISM: abundances — ISM: individual (NGC 7023) — reflection nebulae — stars: individual (HD 200775) — stars: pre-main-sequence — radio lines: ISM 


\section{Introduction}

Although $\mathrm{CO}$ is the most abundant interstellar molecule after $\mathrm{H}_{2}$, its corresponding ion, $\mathrm{CO}^{+}$, is expected to have very low abundance in molecular clouds. The reason is that $\mathrm{CO}^{+}$is quickly converted into $\mathrm{HCO}^{+}$by reactions with $\mathrm{H}_{2}$. Only in the hot layers of photon-dominated regions (PDRs) where a significant fraction of hydrogen is still in atomic form, the $\mathrm{CO}^{+}$abundance becomes significant. Chemical models (Sternberg and Dalgarno 1995) predict that for the PDRs associated with massive stars $\left(\mathrm{n} \sim 10^{6} \mathrm{~cm}^{-3}, \mathrm{G}_{\circ} \sim 210^{5}\right.$ in units of Habing field) the $\mathrm{CO}^{+} / \mathrm{HCO}^{+}$abundance ratio is $\sim 0.05$ at a visual extinction lower than $1.5 \mathrm{mag}$, but decreases by more than 2 orders of magnitude when the extinction increases above 3-5 mag. Based on its chemical behavior, they proposed the $\mathrm{CO}^{+} / \mathrm{HCO}^{+}$ ratio as a tracer of the $\mathrm{HI} / \mathrm{H}_{2}$ transition layer in PDRs.

$\mathrm{CO}^{+}$was tentatively detected for the first time by Erickson et al. 1981 toward OMC-1. Later, Latter, Walker and Maloney 1993 detected $\mathrm{CO}^{+}$in the well-known PDR M17SW and the planetary nebula NGC 7027. More recently, $\mathrm{CO}^{+}$has also been detected in the Orion Bar (Störzer, Stutzki and Sternberg 1995). But so far, all the detections of $\mathrm{CO}^{+}$have been made toward the interfaces between the molecular cloud and the HII regions around massive O stars. Störzer, Stutzki and Sternberg 1995 failed to detect $\mathrm{CO}^{+}$toward the reflection nebula S140. They propose that the large densities and intense UV fields associated with massive $\mathrm{O}$ stars are required to form $\mathrm{CO}^{+}$column densities $\geq 10^{11} \mathrm{~cm}^{-2}$. We present the detection of $\mathrm{CO}^{+}$toward the reflection nebula NGC 7023, which is illuminated by a Be star. Although in this region the incident UV field is, $G_{\circ} \sim 10^{3}$ (in units of Habing field), and densities are, $\mathrm{n} \sim 10^{5} \mathrm{~cm}^{-3}$, we have observed a $\mathrm{CO}^{+}$column density of $\sim 310^{11} \mathrm{~cm}^{-2}$. Furthermore, the spatial-velocity distribution of the $\mathrm{CO}^{+}$emission shows that $\mathrm{CO}^{+}$is only located within the $\mathrm{HI} / \mathrm{H}_{2}$ transition layer of this PDR. 


\section{Observations and Results}

In Plate L1, we show the integrated intensity map of the $\mathrm{HCO}^{+} 1 \rightarrow 0$ (thin dark contours) and the HI column density map (grey scale) of the reflection nebula NGC 7023 (Fuente et al. 1993 and Fuente et al. 1996). The illuminating star, HD 200775, is located in a cavity of the parent cloud delimited by dense walls (Fuente et al. 1993 and references therein). Dense PDRs are found on the surfaces of these walls. In particular, an intense HI clump appears $\approx 40 \mathrm{NW}$ from the star position, at the edge of the bulk of the molecular emission (see Plate L1). Interferometric observations of the $\mathrm{J}=1 \rightarrow 0$ line of $\mathrm{HCO}^{+}$showed the existence of several high density $\mathrm{HCO}^{+}$filaments within this clump (Fuente et al. 1996). The two most intense filaments are also shown in Plate L1. We have searched for $\mathrm{CO}^{+}$ toward the peak position of these filaments. The coordinates of this position are given in Table 1 and hereafter, we will refer to it as the "PDR peak". Beyond the "PDR peak", our $\mathrm{HCO}^{+}$single-dish data show the existence of several clumps immersed in the molecular cloud. We have also searched for $\mathrm{CO}^{+}$toward the molecular clump closest to the "PDR peak" (Plate L1). The coordinates of this position are also given in Table 1 and hereafter, we will refer to it as the "Molecular Peak".

$\mathrm{CO}^{+}$has a ${ }^{2} \Sigma$ ground electronic state in which each rotational level is split in two fine structure levels with $\mathrm{J}=\mathrm{N} \pm 1 / 2$. The $\mathrm{N}=1 \rightarrow 0$ rotational line is heavily obscured by the $\mathrm{O}_{2}$ line at $118 \mathrm{GHz}$ and cannot be observed from ground-based telescopes. The most intense transitions of the $\mathrm{N}=2 \rightarrow 1$ rotational spectrum are $\mathrm{N}=2 \rightarrow 1 \mathrm{~J}=5 / 2 \rightarrow 3 / 2$ at $236062.553 \mathrm{MHz}$ and $\mathrm{N}=2 \rightarrow 1 \mathrm{~J}=3 / 2 \rightarrow 1 / 2$ at $235789.641 \mathrm{MHz}$. In the optically thin limit, the intensity ratio $\mathrm{I}(235.789) / \mathrm{I}(236.062)$ is 0.55 (Sastry et al. 1981). Both line frequencies were covered by the receiver band. Unfortunate, the most intense line is blended with the $5_{-2} \rightarrow 4_{-2}$ and $5_{2} \rightarrow 4_{2}{ }^{13} \mathrm{CH}_{3} \mathrm{OH} \mathrm{E}$ lines (see Blake et al. 1984). In order to determine an upper limit to the ${ }^{13} \mathrm{CH}_{3} \mathrm{OH}$ emission we have observed the $5_{1} \rightarrow 4_{1}$ methanol line toward the PDR peak. To 
determine accurate $\mathrm{CO}^{+}$column densities, it is necessary to have accurate estimates of the hydrogen density. For this aim, maps of about 20 " $\times 20$ " with a spacing of 5 " were carried out around the studied positions in the $\mathrm{CS} \mathrm{J}=2 \rightarrow 1,3 \rightarrow 2$ and $5 \rightarrow 4$ lines. Furthermore, the $\mathrm{H}^{13} \mathrm{CO}^{+} \mathrm{J}=1 \rightarrow 0$ (toward both positions) and $3 \rightarrow 2$ (only toward the molecular peak) have also been observed.

The observations were carried out in 1995 December and 1996 May using the 30-m telescope. The observational procedure was position switching with a fixed reference 30' East from the star. Pointing was checked every two hours using strong continuum sources (NGC 7027, K3-50A, NGC 7538), and the rms of pointing errors was less than 2". The forward and main beam efficiencies were 0.92 and 0.75 at $90 \mathrm{GHz}, 0.90$ and 0.52 at 145 $\mathrm{GHz}$, and 0.86 and 0.37 at $236-260 \mathrm{GHz}$ respectively. The temperature scale is main beam temperature. The HPBW of the telescope was 27" at $90 \mathrm{GHz}, 16$ " at $145 \mathrm{GHz}$ and 10" at $236 \mathrm{GHz}$. Typical system temperatures (in $\mathrm{T}_{M B}$ ) were $300 \mathrm{~K}$ at $90 \mathrm{GHz}, 600 \mathrm{~K}$ at 145 $\mathrm{GHz}, 1300 \mathrm{~K}$ at $236 \mathrm{GHz}$ and 3400 at $260 \mathrm{GHz}$. All the lines have been observed with a frequency resolution of $80 \mathrm{kHz}\left(\sim 0.1 \mathrm{~km} \mathrm{~s}^{-1}\right.$ at $\left.236 \mathrm{GHz}\right)$.

EDITOR: PLACE TABLE 1 HERE.

\subsection{PDR peak}

The $\mathrm{CO}^{+} \mathrm{N}=2 \rightarrow 15 / 2 \rightarrow 3 / 2$ and $3 / 2 \rightarrow 1 / 2$ lines have been detected toward the PDR peak with a signal to noise ratio of 10 and 7 respectively (see Plate L1). The observational parameters are shown in Table 1 . The detection of both lines make very unlikely a possible misidentification. We are not aware of possible line contamination for the transition at 235789.64 MHz. The only possible line contamination comes from the $5_{-2} \rightarrow 4_{-2}$ and $5_{2} \rightarrow 4_{2}$

${ }^{13} \mathrm{CH}_{3} \mathrm{OH}$ E lines whose rest frequency is less than $0.5 \mathrm{MHz}$ from the $\mathrm{CO}^{+}$line at 236062.55 
$\mathrm{MHz}$ (see Blake et al. 1984). Since the observed linewidths of the two lines of $\mathrm{CO}^{+}$are the same, it seems that the line at $236062.55 \mathrm{MHz}$ is very unlikely contaminated by ${ }^{13} \mathrm{CH}_{3} \mathrm{OH}$ lines. To check for possible contamination, we have estimated an upper limit to the emission of the ${ }^{13} \mathrm{CH}_{3} \mathrm{OH}$ lines from the observed $\mathrm{J}_{K}=5_{1} \rightarrow 4_{1}$ methanol line. The excitation conditions and the line strength for this line are very similar to those of the contaminating ${ }^{13} \mathrm{CH}_{3} \mathrm{OH}$ lines (Anderson, De Lucia and Herbst 1990). Assuming a linewidth of $2 \mathrm{kms}^{-1}$, we have obtained a $3 \sigma$ upper limit of $0.2 \mathrm{~K} \mathrm{kms}^{-1}$ to the integrated intensity emission of the methanol line (see Table 1). For an isotopic ratio, $\mathrm{CH}_{3} \mathrm{OH} /{ }^{13} \mathrm{CH}_{3} \mathrm{OH} \sim 40$, this would imply an upper limit of $0.005 \mathrm{~K} \mathrm{kms}^{-1}$ to the integrated intensity emission of each ${ }^{13} \mathrm{CH}_{3} \mathrm{OH}$ line. Since there are two ${ }^{13} \mathrm{CH}_{3} \mathrm{OH}$ lines blended, these lines could contribute to our $\mathrm{CO}^{+}$detection at $236.062 \mathrm{GHz}$ with, at most, an integrated intensity of $0.01 \mathrm{~K} \mathrm{~km}$ $\mathrm{s}^{-1}$. This is only $4 \%$ of the observed integrated intensity emission at $236.062 \mathrm{GHz}$, and it is within the observational errors (see Table 1). We, therefore, conclude that the emission detected at 236.062 and $235.789 \mathrm{GHz}$ is due to $\mathrm{CO}^{+}$.

A striking result of our data is that the $\mathrm{CO}^{+}$lines have linewidths much larger than those of $\mathrm{CS}$ and $\mathrm{H}^{13} \mathrm{CO}^{+}$. The interferometric $\mathrm{HCO}^{+}$filaments detected toward the PDR peak are characterized by having different velocities. The four well detected filaments are centered at radial velocities of $1.9,2.4,2.8$ and $4 \mathrm{~km} \mathrm{~s}^{-1}$, and the one tentatively detected is centered at $5.8 \mathrm{kms}^{-1}$. One of these filaments, $2.4 \mathrm{kms}^{-1}$, is very likely embedded in the molecular cloud, but most of the others, 2.8, 4.0 and $5.8 \mathrm{~km} \mathrm{~s}^{-1}$, seem to be immersed in the atomic medium. The situation is less clear for the filament at $1.9 \mathrm{kms}^{-1}$ that seems to be part of a weak and extended molecular component (Fuente et al. 1996). Therefore, a gradient in the chemical composition of the $\mathrm{HCO}^{+}$filaments is expected depending upon the local visual extinction toward the exciting star. $\mathrm{CS}$ and $\mathrm{H}^{13} \mathrm{CO}^{+}$present narrow lines centered at $2.4 \mathrm{kms}^{-1}$, i.e., the velocity of the filament immersed in the bulk of the molecular cloud. Only $\mathrm{HCO}^{+}$and $\mathrm{CO}^{+}$present emission at the velocities of the filaments 
immersed in the atomic medium. From the comparison of the spectra of the $\mathrm{H}^{13} \mathrm{CO}^{+}$, $\mathrm{HCO}^{+}$and $\mathrm{CO}^{+}$lines, it is clear that there exists a gradient in the $\mathrm{CO}^{+} / \mathrm{HCO}^{+}$abundance ratio as a function of velocity, i.e., as a function of the visual extinction from the star (see Plate L1). To determine this gradient, we have estimated the $\mathrm{CO}^{+} / \mathrm{HCO}^{+}$abundance ratio in three different velocity intervals, $0-1.6 \mathrm{~km} \mathrm{~s}^{-1}, 1.6-3.2 \mathrm{~km} \mathrm{~s}^{-1}$, and $3.2-6 \mathrm{~km} \mathrm{~s}^{-1}$.

$\mathrm{CO}^{+}$column densities have been estimated using the LTE approximation. Assuming $\mathrm{T}_{K}=40 \mathrm{~K}$ (see Fuente et al. 1993) we derived from CS data a hydrogen density of $\sim 3.5$ $10^{5} \mathrm{~cm}^{-3}$ for the component at $2.4 \mathrm{~km} \mathrm{~s}^{-1}$. Similar densities were obtained for the other filaments from the interferometric $\mathrm{HCO}^{+}$data (Euente et al. 1996). Using a LVG code and assuming $\mathrm{T}_{k}=40 \mathrm{~K}$ and $\mathrm{n}=3.510^{5} \mathrm{~cm}^{-3}$, we estimate $\mathrm{T}_{\text {rot }}=10 \mathrm{~K}$ for $\mathrm{HCO}^{+}$. Since $\mathrm{HCO}^{+}$and $\mathrm{CO}^{+}$have similar dipole moments and rotational constants $(\mu=2.77 \mathrm{D}$ for $\mathrm{CO}^{+}$and $3.91 \mathrm{D}$ for $\mathrm{HCO}^{+}$), we assume the same rotational temperature for $\mathrm{CO}^{+}$. In Table 2 we show the derived $\mathrm{HCO}^{+}, \mathrm{H}^{13} \mathrm{CO}^{+}$and $\mathrm{CO}^{+}$column densities. From these estimates, we have determined that the $\mathrm{CO}^{+} / \mathrm{HCO}^{+}$abundance ratio is a factor of 10 larger for the filaments immersed in the atomic region than for the filaments embedded in the molecular cloud. This gradient in the $\mathrm{CO}^{+} / \mathrm{HCO}^{+}$ratio cannot be due to an opacity effect. The $\mathrm{I}\left(\mathrm{CO}^{+} 235.789\right) / \mathrm{I}\left(\mathrm{CO}^{+} 236.062\right)$ ratio is consistent with optically thin emission (within the observational errors) for all the velocity intervals (see Table 2). Though consistent with optically thin emission, our data suggest that the opacities of the $\mathrm{CO}^{+}$lines could be larger for the velocities $3.2-6.0 \mathrm{~km} \mathrm{~s}^{-1}$ than for $1.6-3.2 \mathrm{~km} \mathrm{~s}^{-1}$. In this case, the $\mathrm{CO}^{+}$column density would be slightly underestimated for the velocity interval $3.2-6.0 \mathrm{~km} \mathrm{~s}^{-1}$, and the derived $\mathrm{CO}^{+} / \mathrm{HCO}^{+}$ratio would be a lower limit to the actual value of the $\mathrm{CO}^{+} / \mathrm{HCO}^{+}$ ratio for this interval. Therefore, although we are aware of the uncertainties involved in column density estimates, we think that the observed gradient in the $\mathrm{CO}^{+} / \mathrm{HCO}^{+}$ ratio (a factor of 10) is significant, and it is in agreement with the expected behavior of the $\mathrm{CO}^{+} / \mathrm{HCO}^{+}$ratio, where $\mathrm{CO}^{+}$formation is restricted to a narrow range of visual 
extinctions $\mathrm{A}_{v}<2 \mathrm{mag}$. The visual extinction at the surface of the filament at $2.4 \mathrm{~km} \mathrm{~s}^{-1}$ must be $>1$ mag to be immersed in a mainly molecular medium, while for the filaments immersed in a mainly atomic medium, the visual extinction must be $<1$ mag. Assuming a $\mathrm{HCO}^{+}$fractional abundance of $410^{-10}$ (Fuente et al. 1996), the $\mathrm{CO}^{+}$fractional abundance is $\sim 410^{-11}$ in the filaments immersed in the atomic medium. $\mathrm{CO}^{+}$fractional abundances $\sim 10^{-11}$ are also derived from the $\mathrm{CO}^{+}$data reported by Störzer, Stutzki and Sternberg 1995 and Latter, Walker and Maloney 1993, toward M17SW and the Orion Optical Bar. Although the physical conditions and incident UV field are different (see Section 3), the $\mathrm{CO}^{+}$fractional abundance in NGC 7023 is similar to that found at the edges of the HII regions around massive stars.

\section{EDITOR: PLACE TABLE 2 HERE.}

\subsection{Molecular peak}

We have not detected $\mathrm{CO}^{+}$toward the molecular peak. Assuming a linewidth of $1 \mathrm{~km}$ $\mathrm{s}^{-1}$ (a typical linewidth for the molecular cloud), we obtain an upper limit to the integrated intensity of the $\mathrm{CO}^{+}$line at $236.062 \mathrm{GHz}$ of $0.03 \mathrm{~K} \mathrm{kms}^{-1}$. Assuming a kinetic temperature of $\mathrm{T}_{K}=15 \mathrm{~K}$ (Fuente et al. 1990), we estimate a density of $10^{5} \mathrm{~cm}^{-3}$ from our CS data. This density is high enough to excite the $\mathrm{CO}^{+}$lines. In fact, the excitation conditions required for the $\mathrm{H}^{13} \mathrm{CO}^{+} \mathrm{J}=3 \rightarrow 2$ line are comparable to those required for the $\mathrm{CO}^{+} \mathrm{N}=2 \rightarrow 1$ $\mathrm{J}=5 / 2 \rightarrow 3 / 2$ and $3 / 2 \rightarrow 1 / 2$ lines, and the $\mathrm{H}^{13} \mathrm{CO}^{+} \mathrm{J}=3 \rightarrow 2$ line has been detected with an intensity of $1.04 \mathrm{~K}$. Therefore, the lack of detection of $\mathrm{CO}^{+}$toward the molecular peak is not due to the excitation conditions in this region. With the same assumptions as for the

$\mathrm{PDR}$ peak, the upper limit to the $\mathrm{CO}^{+}$column density is $4.510^{10} \mathrm{~cm}^{-2}$. Assuming $\mathrm{n}=10^{5}$ $\mathrm{cm}^{-3}$ and $\mathrm{T}_{K}=15 \mathrm{~K}$, we estimate a $\mathrm{H}^{13} \mathrm{CO}^{+}$column density of $810^{11} \mathrm{~cm}^{-2}$. This means a 
$\mathrm{CO}^{+} / \mathrm{HCO}^{+}$ratio of $<0.001$. Therefore, the $\mathrm{CO}^{+} / \mathrm{H}^{13} \mathrm{CO}^{+}$ratio is at least 100 times lower in the molecular peak than in the filaments immersed in the atomic medium. Assuming a $\mathrm{HCO}^{+}$abundance of $410^{-10}$, we obtain a fractional abundance of $\mathrm{CO}^{+}$of $<510^{-13}$ in the molecular peak.

\section{Summary and Discussion}

We have detected, for the first time, $\mathrm{CO}^{+}$in a PDR associated with a Be star. This region is very different from the massive star forming regions where $\mathrm{CO}^{+}$had been detected thus far. First of all, since the ionization potential of $\mathrm{CO}$ is larger than $13.6 \mathrm{eV}$, a $\mathrm{Be}$ star does not produce a significant number of photons capable to ionize CO. Furthermore, the intensity of the UV field and the density around this star, $G_{\circ} \sim 10^{3}$ ( in units of Habing field), and densities of $\sim 10^{5} \mathrm{~cm}^{-3}$, are very different from those around massive $\mathrm{O}$ stars where $G_{\circ} \sim 10^{5}$ and $\mathrm{n} \geq 10^{6} \mathrm{~cm}^{-3}$. Chemical models predict that $\mathrm{CO}^{+}$column densities decrease sharply for UV fields $<10^{5}$, and densities $<10^{6} \mathrm{~cm}^{-3}$ (Störzer, Stutzki and Sternberg 1995). Even for the conditions prevailing in massive star forming regions, chemical models fail to predict the large $\mathrm{CO}^{+}$column densities observed toward them. To solve this problem, some authors have suggested that the direct photoionization of CO might be a non-negligible formation mechanism of $\mathrm{CO}^{+}$in these regions (Jansen et al. 1995, Black, Latter and Maloney 1996). We have estimated a $\mathrm{CO}^{+}$column density of $\sim 3$ $10^{11} \mathrm{~cm}^{-2}$ toward the PDR peak in NGC 7023. Our results show that large $\mathrm{CO}^{+}$column

densities can be produced even with UV fields of just a few $10^{3}$ and densities of around $10^{5}$ $\mathrm{cm}^{-3}$. Since the peak $\mathrm{CO}^{+}$abundance in NGC $7023\left(\sim 410^{-11}\right)$ is similar to that found in massive star forming regions, our data suggest that the direct photoionization of CO is not a significant formation mechanism for $\mathrm{CO}^{+}$. 
We are grateful to the technical staff of Pico de Veleta for their support during the observations. We are also grateful to Dr. R. Gaume for his careful reading of the manuscript. This work has been partially supported by the Spanish DGICYT under grant number PB93-0048. 


\section{REFERENCES}

Anderson, T., De Lucia, C., Herbst, E., 1990, ApJS, 72, 797

Black, J. H., Latter, W. B., Maloney, P. R., 1996, "Abundance and excitation of reactive molecular ions", Molecules in Astrophysics: Probes \& Processes, IAU sumposium 178 Abstract Book (1 - 5 July 1996, Leiden, The Netherlands), edited by D.J. Jansen, M. R. Hogerheijde, \& E. F. van Dishoeck

Blake, G. A., Sutton, E. C., Masson, C. R., Phillips, T. G., Herbst, E., Plummer, G. M., De Lucia, F. C., 1984, ApJ, 286, 586

Erickson, N. R., Snell, R. L., Loren, R. B., Mundy, L., Plambeck, R. L., 1981, ApJ, 245, L83

Fuente, A., Martín-Pintado, J., Cernicharo, J., Bachiller, R., 1990, A\&A, 237, 471

Fuente, A., Martín-Pintado, J., Cernicharo, J., Bachiller, R., 1993, A\&A, 276, 476

Fuente, A., Martín-Pintado, J., Neri, R., Rogers, C., Moriarty-Schieven, G., 1996, A\&A, 310,286

Jansen, D. J., Spaans, M., Hogerheijde, M. R., van Dishoeck, E. F., 1995, A\&A, 303, 541

Latter, W. B., Walker, C. K., Maloney, P. R., , ApJ, 419, L97

Sastry, K. V. L. N., Helminger, P., Herbst, E., De Lucia, F. C., 1981, ApJ, 250, L91

Sternberg, A., Dalgarno, A., 1995, ApJS, 99, 565

Störzer, H., Stutzki, J., Sternberg, A., 1995, A\&A, 296, L9 
Fig. 1. - Left panel shows the integrated intensity map of the $\mathrm{HCO}^{+} \mathrm{J}=1 \rightarrow 0$ line carried out with the 30-m telescope toward NGC 7023 (solid contours) superposed to the HI column density image obtaining after combining VLA and DRAO data (grey scale) (Fuente et al. 1993, Fuente et al. 1996). $\mathrm{HCO}^{+}$contours are 0.8 to 7.2 by $0.8 \mathrm{~K} \mathrm{kms}^{-1}$. The numbers in the wedge are in units of $10^{20} \mathrm{~cm}^{-2}$. The star indicates the position of HD 200775, the white triangle indicates the PDR peak and the filled black square, the molecular peak. The $\mathrm{HCO}^{+}$ molecular filaments as observed with the IRAM PdB interferometer are the thick contours, black is the filament at $2.4 \mathrm{kms}^{-1}$ and white the one at $4 \mathrm{kms}^{-1}$ (Fuente et al. 1996). On the right we show (from top to down) the spectra of the $\mathrm{HCO}^{+} \mathrm{J}=1 \rightarrow 0, \mathrm{H}^{13} \mathrm{CO}^{+} \mathrm{J}=1 \rightarrow 0$ $\mathrm{CO}^{+} \mathrm{N}=2 \rightarrow 1 \mathrm{~J}=5 / 2 \rightarrow 3 / 2$ and $\mathrm{J}=3 / 2 \rightarrow 1 / 2$ lines toward the molecular peak and the PDR peak. Several channels of the original $\mathrm{CO}^{+}$spectra have been averaged. 
Table 1: Observational parameters

\begin{tabular}{|c|c|c|c|c|c|c|}
\hline Position & Molecule & $\begin{array}{c}\text { Frequency } \\
(\mathrm{MHz})\end{array}$ & $\begin{array}{c}\int T_{M B} d v \\
\left(\mathrm{~K} \mathrm{~km} \mathrm{~s}^{-1}\right)\end{array}$ & $\begin{array}{c}\mathrm{v}_{l s r} \\
\left(\mathrm{~km} \mathrm{~s}^{-1}\right)\end{array}$ & $\begin{array}{c}\Delta v \\
\left(\mathrm{~km} \mathrm{~s}^{-1}\right)\end{array}$ & $\begin{array}{l}\mathrm{T}_{M B} \\
(\mathrm{~K})\end{array}$ \\
\hline PDR peak & $\mathrm{CO}^{+}$ & 235789.64 & $0.17(0.03)$ & $3.4(0.2)$ & $2.1(0.4)$ & 0.076 \\
\hline R.A.: $21^{h} 01^{m} 32^{s} .6$ & $\mathrm{CO}^{+}$ & 236062.55 & $0.23(0.02)$ & $2.7(0.1)$ & $2.1(0.4)$ & 0.104 \\
\hline Dec: $68^{\circ} 10^{\prime} 27^{\prime \prime}$ & $\mathrm{CH}_{3} \mathrm{OH}$ & 239746.25 & $<0.2^{\mathrm{a}}$ & & & \\
\hline \multirow[t]{4}{*}{$(2000)$} & CS & 97980.97 & $0.92(0.04)$ & $2.3(0.1)$ & $0.7(0.1)$ & 1.31 \\
\hline & $\mathrm{CS}^{\mathrm{b}}$ & 146969.05 & $1.38(0.02)$ & $2.3(0.1)$ & $0.7(0.1)$ & 1.85 \\
\hline & $\mathrm{CS}^{\mathrm{c}}$ & 244935.61 & $0.79(0.03)$ & $2.3(0.1)$ & $0.5(0.1)$ & 2.73 \\
\hline & $\mathrm{H}^{13} \mathrm{CO}^{+}$ & 86754.33 & $0.34(0.04)$ & $2.38(0.04)$ & $0.5(0.1)$ & 0.61 \\
\hline Mol. peak & $\mathrm{CO}^{+}$ & 235789.64 & $<0.03^{\mathrm{d}}$ & & & \\
\hline R.A.: $21^{h} 01^{m} 31^{s} .6$ & $\mathrm{CO}^{+}$ & 236062.55 & $<0.03^{\mathrm{d}}$ & & & \\
\hline Dec: $68^{\circ} 11^{\prime} 12^{\prime \prime}$ & CS & 97980.97 & $1.02(0.02)$ & $2.6(0.1)$ & $0.7(0.1)$ & 1.36 \\
\hline \multirow[t]{4}{*}{$(2000)$} & $\mathrm{CS}^{\mathrm{b}}$ & 146969.05 & $0.64(0.02)$ & $2.7(0.1)$ & $0.7(0.1)$ & 0.9 \\
\hline & CS & 244935.61 & $<0.04^{\mathrm{d}}$ & & & \\
\hline & $\mathrm{H}^{13} \mathrm{CO}^{+}$ & 86754.33 & $1.10(0.01)$ & $2.8(0.1)$ & $0.7(0.1)$ & 1.46 \\
\hline & $\mathrm{H}^{13} \mathrm{CO}^{+}$ & 260255.48 & $0.44(0.01)$ & $3.0(0.1)$ & $0.4(0.1)$ & 1.04 \\
\hline
\end{tabular}

${ }^{a} 3 \sigma$ upper limit assuming a linewidth of $2 \mathrm{kms}^{-1}$

${ }^{b}$ Spectrum after degrading the angular resolution of the CS $3 \rightarrow 2$ map to have that of the CS $2 \rightarrow 1$ map.

${ }^{c}$ The same as b but for the CS $5 \rightarrow 4$ map

${ }^{d} 3 \sigma$ upper limit assuming a linewidth of $1 \mathrm{kms}^{-1}$ 
Table 2: Integrated intensities, column densitites and abundance estimates toward the PDR peak per velocity interval

\begin{tabular}{lccc} 
& $0-1.6 \mathrm{kms}^{-1}$ & $1.6-3.2 \mathrm{kms}^{-1}$ & $3.2-6.0 \mathrm{kms}^{-1}$ \\
\hline $\mathrm{I}\left(\mathrm{HCO}^{+} \mathrm{J}=1 \rightarrow 0\right)\left(\mathrm{K} \mathrm{kms}^{-1}\right)$ & $0.59(0.02)^{\mathrm{a}}$ & $4.67(0.02)$ & $1.10(0.03)$ \\
$\mathrm{I}\left(\mathrm{H}^{13} \mathrm{CO}^{+} \mathrm{J}=1 \rightarrow 0\right)\left(\mathrm{K} \mathrm{kms}^{-1}\right)$ & $0.11(0.04)$ & $0.34(0.04)$ & $<0.15$ \\
$\mathrm{I}\left(\mathrm{CO}^{+} \mathrm{N}=2 \rightarrow 1 \mathrm{~J}=5 / 2 \rightarrow 3 / 2\right)\left(\mathrm{K} \mathrm{kms}^{-1}\right)$ & $0.02(0.01)$ & $0.14(0.01)$ & $0.07(0.02)$ \\
$\mathrm{I}\left(\mathrm{CO}^{+} \mathrm{N}=2 \rightarrow 1 \mathrm{~J}=3 / 2 \rightarrow 1 / 2\right)\left(\mathrm{K} \mathrm{kms}^{-1}\right)$ & $<0.06$ & $0.08(0.02)$ & $0.09(0.03)$ \\
$\frac{I\left(C O^{+} N=2 \rightarrow 1 J=3 / 2 \rightarrow 1 / 2\right)}{I\left(C O^{+}=2 \rightarrow 1 J=5 / 2 \rightarrow 3 / 2\right)}$ & & $0.6(0.2)$ & $1.3(0.8)$ \\
$\mathrm{N}\left(\mathrm{HCO}^{+}\right)\left(\mathrm{cm}^{-2}\right)$ & $510^{11}$ & $410^{12}$ & $910^{11}$ \\
$\mathrm{~N}\left(\mathrm{H}^{13} \mathrm{CO}^{+}\right)\left(\mathrm{cm}^{-2}\right)$ & $810^{10}$ & $310^{11}$ & \\
$\mathrm{~N}\left(\mathrm{CO}^{+}\right)\left(\mathrm{cm}^{-2}\right)$ & $310^{10}$ & $210^{11}$ & $110^{11}$ \\
$\frac{N\left(C O^{+}\right)}{N\left(H C O^{+}\right)}$ & $0.01^{\mathrm{b}}$ & $0.02^{\mathrm{b}}$ & 0.11 \\
$\mathrm{X}\left(\mathrm{CO}^{+}\right)^{\mathrm{c}}$ & $410^{-12}$ & $810^{-12}$ & $410^{-11}$ \\
\hline
\end{tabular}

${ }^{a}$ The number in parenthesis is $\sigma$

${ }^{b}$ In these cases, the $\mathrm{HCO}^{+}$column density has been estimated from $\mathrm{H}^{13} \mathrm{CO}^{+}$data assuming an isotopic ratio of 40

${ }^{c}$ Assuming $\mathrm{X}\left(\mathrm{HCO}^{+}\right) \sim 410^{-10}$ 


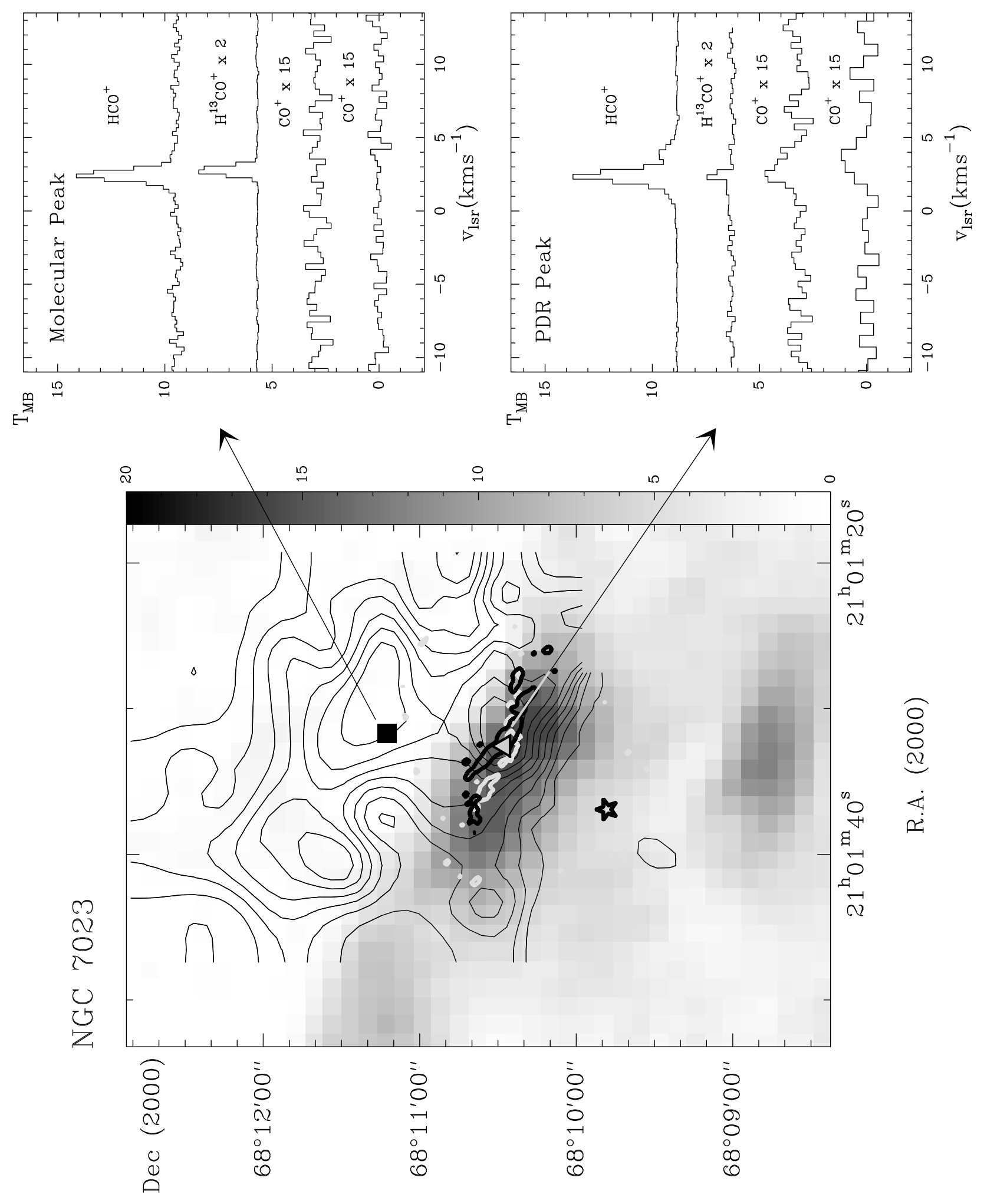

